\title{
Therapeutic Effects of Benralizumab Assessed in Patients with Severe Eosinophilic Asthma: Real-Life Evaluation Correlated with Allergic and Non-Allergic Phenotype Expression
}

This article was published in the following Dove Press journal:

Journal of Asthma and Allergy

\author{
Corrado Pelaia (D) \\ Claudia Crimi (iD) \\ Alida Benfante 3 \\ Maria Filomena Caiaffa ${ }^{4}$ \\ Cecilia Calabrese ${ }^{5}$ \\ Giovanna Elisiana Carpagnano ${ }^{6}$ \\ Domenico Ciotta Jnr (iD) \\ Maria D'Amato ${ }^{8}$ \\ Luigi Macchia9 \\ Santi Nolasco (iD) ${ }^{2}$ \\ Girolamo Pelaia (D) \\ Simona Pellegrino ${ }^{7}$ \\ Nicola Scichilone (iD ${ }^{3}$ \\ Giulia Scioscia $^{10}$ \\ Giuseppe Spadaro " \\ Giuseppe Valenti ${ }^{12}$ \\ Alessandro Vatrella iD $^{7}$ \\ Nunzio Crimi ${ }^{2}$ \\ 'Department of Health Sciences, University “Magna \\ Graecia" of Catanzaro, Catanzaro, Italy; ${ }^{2}$ Department of \\ Clinical and Experimental Medicine, University of Catania, \\ Catania, Italy; ${ }^{3}$ Department of Biomedicine and Internal and \\ Specialistic Medicine, University of Palermo, Palermo, Italy; \\ ${ }^{4}$ Allergology and Clinical Immunology Unit, University of \\ Foggia, Foggia, Italy; ${ }^{5}$ Department of Translational Medical \\ Sciences, University of Campania “Luigi Vanvitelli”, Foggia, \\ Italy; ${ }^{6}$ Department of Basic Medical Sciences, Neuroscience \\ and Sense Organs, Section of Respiratory Disease, \\ University "Aldo Moro" of Bari, Bari, Italy; ${ }^{7}$ Department of \\ Medicine, Surgery and Dentistry, University of Salerno, \\ Salerno, Italy; ${ }^{8}$ Division of Pneumology, "V. Monaldi" \\ University Hospital, Naples, Italy; ${ }^{9}$ Allergology and Clinical \\ Immunology Unit, University “Aldo Moro" of Bari, Bari, \\ Italy; ${ }^{10}$ Department of Medical and Surgical Sciences, \\ Institute of Respiratory Diseases, University of Foggia, \\ Foggia, Italy; "'Allergology and Immunology Unit, University \\ "Federico II" of Naples, Naples, Italy; ${ }^{12}$ Allergology and \\ Pulmonology Unit, Provincial Outpatient Center of \\ Palermo, Palermo, Italy
}

Correspondence: Corrado Pelaia Department of Health Sciences, University “Magna Graecia” of Catanzaro, Catanzaro, Italy Tel + 39 096I 3647007

Email pelaia.corrado@gmail.com
Background: Benralizumab can be utilized as add-on biological treatment of severe eosinophilic asthma. However, so far only a few real-life studies have been published with regard to the use of this anti-IL-5 receptor humanized monoclonal antibody.

Objective: The primary aim of this multicenter observational investigation has been to assess the therapeutic effects of benralizumab in patients with severe uncontrolled, corticosteroid refractory eosinophilic asthma. The secondary objective was to evaluate the efficacy of benralizumab with regard to positive or negative skin prick test (SPT).

Methods: Clinical, functional, and laboratory parameters were evaluated in order to verify the therapeutic actions of benralizumab in atopic and non atopic subjects with difficult-totreat eosinophilic asthma. Moreover, a comparative evaluation was carried out in relation to the presence or absence of SPT positivity.

Results: After 6 months of add-on biological therapy with benralizumab, our 111 patients experienced a marked improvement of their severe eosinophilic asthma, expressed by significant changes in asthma exacerbation rate, prednisone intake, daily use of shortacting $\beta_{2}$-adrenergic agonists (SABA), asthma control test (ACT) score, asthma quality of life questionnaire (AQLQ) score (56 patients), forced expiratory volume in one second $\left(\mathrm{FEV}_{1}\right)$, forced vital capacity (FVC), blood eosinophil count, blood basophil count (59 patients), and fractional exhaled nitric oxide (FeNO) levels (39 patients). In addition, significantly more effective outcomes were detected in patients with positive SPT, when compared to subjects with negative SPT, only in regard to asthma exacerbation number, ACT score, and daily SABA utilization. No significant correlation was found between serum IgE concentrations and each of all measured parameters.

Conclusion and Clinical Relevance: Taken together, the results of this real-world study indicate that in both allergic and non-allergic subjects benralizumab can be used as a valuable pharmacotherapeutic option for add-on biological therapy of severe eosinophilic asthma, regardless of SPT positivity or negativity.

Keywords: severe eosinophilic asthma, allergic and non-allergic phenotypes, asthma exacerbations, IL-5 receptor, benralizumab

\section{Introduction}

Over 300 million people worldwide suffer from asthma, a chronic respiratory disorder commonly shaped by variable airflow limitation and airway hyperresponsiveness, underpinned by predominant inflammatory features consisting of several 
phenotypes. ${ }^{1-7}$ Among the latter, bronchial eosinophilia characterizes many forms of both allergic and nonallergic asthma, and high eosinophil numbers can be detected in blood and induced sputum from patients experiencing recurrent disease exacerbations and severe bronchoconstriction. ${ }^{8}$ Airway eosinophilic infiltration is indeed highly frequent in severe asthma, which is often associated with an inflammatory trait driven by a type- 2 (T2-high) immune response sustained by key pathogenic roles played by $\mathrm{T}$ helper 2 (Th2) lymphocytes and group 2 innate lymphoid cells (ILC2). ${ }^{9-12}$ Either atopic or non atopic phenotypes of asthma can be characterized by eosinophilic inflammation, ${ }^{13}$ which is usually sensitive to corticosteroid therapy. However, severe eosinophilic asthma can be resistant to inhaled corticosteroids (ICS) and also to oral corticosteroids (OCS) because of different mechanisms including an up-regulation of the dysfunctional $\beta$ isoform of glucocorticoid receptor, a p38 MAPK (mitogen-activated protein kinase)-dependent inactivation of glucocorticoid receptor, and an impairment of histone deacetylase function. ${ }^{14-16}$

For patients expressing T2-high phenotypes/endotypes of severe asthma, the step 5 of current GINA (Global Initiative for Asthma) guidelines recommends a maximal medical treatment. ${ }^{17}$ The latter consists of high doses of ICS/LABA (long-acting $\beta_{2}$-adrenergic agonists) combinations, inhaled tiotropium and eventually an oral leukotriene inhibitor, as well as an add-on biological therapy. ${ }^{17}$ Licensed monoclonal antibodies indicated for biological treatment of severe asthma include anti-IgE omalizumab, anti-IL-5 mepolizumab and reslizumab, IL-5 receptor (IL5R) antagonist benralizumab, and dual IL-4/IL-13 receptor blocker dupilumab. ${ }^{18-26}$ In this regard it is noteworthy that many severe asthmatic patients can be responsive to different biologic therapies, ${ }^{27}$ thereby committing physicians to a complex pharmacologic choice. Within such a context, the therapeutic effectiveness of omalizumab in eosinophilic allergic asthma has been convincingly demonstrated along the last two decades by both randomized controlled studies and real-world experiences. ${ }^{18,19}$ Moreover, some post hoc evaluations of recent Phase 3 trials, ${ }^{28-30}$ and also a few real-life observations referring to relatively small numbers of patients with severe asthma, ${ }^{31-38}$ suggest that mepolizumab, benralizumab and dupilumab can be very effective in both allergic and non-allergic asthma.

On the basis of the above considerations, the aim of this real-world multicenter, observational investigation, has been to assess in more than 100 subjects with severe eosinophilic asthma the effects of a 24-week add-on treatment with benralizumab. In particular, the rationale underlying our study refers to the comparative evaluation of benralizumab efficacy in allergic and non-allergic patients, an important aspect which has not been thoroughly addressed by previous trials.

\section{Patients and Methods Study Design and Patient Enrollment}

In 111 patients with severe eosinophilic asthma, a multicenter, real-life study was carried out to evaluate the therapeutic effects of benralizumab, also in relation to allergic/non-allergic phenotype expression. Utilizing a common database regarding severe asthmatic outpatients referring in the last two years to 12 specialized asthma centers located in Southern Italy, the 111 enrolled subjects were consecutively selected on the basis of their treatment with benralizumab. When considering the total number of patients included within this database, the most frequently used drug for add-on biological therapy of severe asthma was omalizumab, followed by mepolizumab and benralizumab.

More than 18 yrs old adult patients, suffering from severe persistent eosinophilic asthma, were recruited. They were on treatment with high dosages of ICS-LABA associations, eventually integrated by the addition of inhaled long-acting muscarinic antagonists (LAMA), and/ or leukotriene inhibitors. In particular, 85 out of the 111 enrolled patients were using LAMA. Moreover, most of these subjects required recurrent courses of OCS therapy, comprehensively lasting at least 6 months/year. However, despite such strong treatments, asthma symptoms and disease exacerbations were very frequent. Indeed, these patients experienced a median number of 4 exacerbations during the 6 months before the first benralizumab administration, and $72 \%$ of recruited subjects were on maintenance therapy with OCS. In regard to ongoing corticosteroid treatment, we carefully verified that all patients were characterized by a high adherence to both ICS and OCS intake during the 6 months prior to the initiation of benralizumab therapy, as well as throughout the 6 months following the first injection of this biologic drug.

Skin prick test (SPT) positivity and negativity were considered in order to identify either allergic or nonallergic patients. SPT was judged as positive when the 
wheal area was equal to or larger than that one caused by histamine. Higher than $100 \mathrm{UI} / \mathrm{mL}$ levels of serum IgE were indicative of an atopic trait. ${ }^{39,40}$ All subjects satisfied the diagnostic criteria of severe uncontrolled asthma, jointly established by the European Respiratory Society (ERS) and the American Thoracic Society (ATS). ${ }^{41}$ The involved asthma centers used a shared database to collect clinical, functional, and biological data. Smoking habit and comorbidities, such as gastro-esophageal reflux disease (GERD), nasal polyposis, bronchiectasis, osteoporosis, anxiety, atopic dermatitis, and obstructive sleep apnea syndrome (OSAS), were investigated prior to beginning add-on therapy with benralizumab. Spirometry was carried out following ATS/ERS guidelines ${ }^{42}$ at baseline, 1 month, 3 months and 6 months after the first dose of benralizumab. Nasal polyposis was detected by nasal endoscopy and/or computerized tomography of paranasal sinuses. Benralizumab was prescribed according to currently used eligibility criteria. In particular, this biological drug was injected subcutaneously at the dosage of $30 \mathrm{mg}$ every 4 weeks for the first three times, and then every 8 weeks.

This observational study satisfied the standards of Good Clinical Practice (GCP) and the principles of the Declaration of Helsinki. In addition, informed consent was obtained from all patients. Our investigation was also conducted according to what stated by the local Ethical Committee of Calabria Region (Catanzaro, Italy; document n. 113-16th April, 2020).

\section{Outcomes and Measurements}

The main aim of this real-life experience was to verify the efficacy of benralizumab in routine clinical practice. Asthma exacerbation rate and daily OCS consumption were assessed at baseline and 6 months after the first benralizumab administration. SABA use, asthma control test (ACT) score, asthma quality of life questionnaire (AQLQ) score, forced expiratory volume in one second $\left(\mathrm{FEV}_{1}\right)$, forced vital capacity (FVC), blood eosinophil numbers, blood basophil counts, and exhaled fraction of nitric oxide (FeNO) levels were measured at baseline, 1 month, 3 months and 6 months after the first benralizumab injection.

The secondary goal was to evaluate the efficacy of benralizumab in relation to SPT positivity or negativity. Moreover, after 6 months of additional therapy with benralizumab, we analyzed the eventual correlation occurring between baseline serum $\operatorname{IgE}$ concentrations and the recorded modifications referring to exacerbation rate, daily prednisone consumption, short-acting $\beta_{2}$-adrenergic agonist (SABA) use, ACT score, AQLQ score, FEV ${ }_{1}$, FVC, blood eosinophil numbers, blood basophil counts, and FeNO levels.

For two hours after benralizumab injection, patients were accurately monitored, because of the possible onset of side effects and adverse reactions. Furthermore, the eventual previous occurrence of unwanted drug effects was investigated each time every patient underwent benralizumab administration.

\section{Statistical Analysis}

Prism Version 8.2.1 (GraphPad Software Inc., San Diego, California) was used to statistically analyze study results. Median values with interquartile range (IQR) were used to express skewed data distributions, while normally distributed data were expressed as mean \pm standard deviation (SD). In consideration of data normality, parametric or non-parametric tests were chosen. The normal distribution of data was assessed by using Anderson-Darling test and Kolmogorov-Smirnov test. When appropriate, variable comparations were performed using Student $t$-test or Mann-Whitney $U$-test. The latter statistical test was also utilized for the secondary study outcome, referring to the comparative evaluation of benralizumab efficacy in patients with either positive or negative SPT. Fisher exact test was applied to compare categorical variables. The association between baseline serum IgE concentrations and changes in clinical, functional and laboratory parameters was investigated using linear regression analysis. Spearman's rank correlation coefficient was assessed. When appropriate, post hoc power analysis was carried out. Statistical significance was set at a threshold of $\mathrm{p}$ value $<0.05$.

\section{Results \\ Patient Features}

Overall, 111 participants (71 females and 40 males) were enrolled in this observational study. The median (IQR) age of our study population was 56 (43-65) years, and the median (IQR) disease duration was 18 years (10-30). Median (IQR) baseline $\mathrm{FEV}_{1}$ was 61\% (49.75-74.25) of predicted value. Median (IQR) body mass index (BMI) was 26.17 (23.14-28.13). Median (IQR) blood eosinophil number was 740.0 cells $/ \mu \mathrm{L}$ (200.0-1097), and median (IQR) blood basophil count was 44.0 cells $/ \mu \mathrm{L}(30.0-86.0)$. Sixty 
(54.1\%) patients were SPT positive, 32 (28.8\%) subjects were smokers or former smokers, 55 (49.5\%) had gastroesophageal reflux disease (GERD), 51 (45.9\%) suffered from nasal polyposis, 27 (24.3\%) had osteoporosis, 21 (18.9\%) complained of anxiety, 13 (11.7\%) had atopic dermatitis, and 11 (9.9\%) suffered from obstructive sleep apnea syndrome (OSAS). Moreover, the presence of bronchiectasis was evidenced in $27(24.3 \%)$ participants. No patient was previously treated with other biologics.

Baseline patient features also characterized on the basis of SPT positivity or negativity, are summarized in Table 1. At baseline, no difference was found between SPT positive and negative patients with regard to AQLQ and ACT scores. No significant difference was detected with regard to either allergic or non-allergic trait, except for disease duration, which resulted to be longer in allergic patients $(p<0.001)$, and atopic dermatitis, that was more common among allergic subjects $(\mathrm{p}<0.01)$; $\mathrm{p}$ values relate to SPT negative versus SPT positive patients. Furthermore, we found that 46 subjects were allergic to dust mites, 38 to pollens, and 20 to pet dander; 32 patients were allergic to more than one aeroallergen. In addition, in 39 (35.1\%) out of the 111 enrolled subjects we measured FeNO, whose baseline median level was 48 parts per billion (ppb) (35-67).

\section{Efficacy of Benralizumab in the Whole Population}

In comparison to the 6-month period preceding the first benralizumab administration, asthma exacerbation rate sharply dropped from $4(3-6)$ to $0(0-0)$ after six months of antiIL-5R $\alpha$ therapy $(\mathrm{p}<0.0001)$ (Figure 1A). This remarkable therapeutic outcome made it possible to lower OCS consumption from $5 \mathrm{mg}(0-12.5)$ to $0 \mathrm{mg}(0-0)(\mathrm{p}<0.0001)$ (Figure 1B). Moreover, with respect to baseline, after 6 months of benralizumab treatment the percentage of subjects needing a daily prednisone intake decreased from $72 \%$ to $20 \%$. Otherwise, no change in ICS dosage was detected throughout the study period (data not shown). Daily SABA inhalations, used as needed rescue medications, decreased from $2(1-4)$ to $0(0-0)(\mathrm{p}<0.0001)$ after 1 month, as well as after 3 and 6 months (Figure $1 \mathrm{C}$ ).

Six months after add-on therapy with benralizumab, ACT score enhanced from a baseline value of 13.31 ( \pm $3.961)$ to $21.88( \pm 2.835)(\mathrm{p}<0.0001)$. This result was already relevant after 1 month, when ACT score was $19.05( \pm 4.334)(\mathrm{p}<0.0001)$, and after 3 months, when ACT score was $21.09( \pm 3.250)(\mathrm{p}<0.0001)$ (Figure
1D). In 56 out of 111 patients, AQLQ score was also examined; it increased from a baseline value of 3.0 (2.$09-4.0)$, to $4.35(3.625-6.39)(\mathrm{p}<0.001), 5.075$ $(4.8-7.0)(\mathrm{p}<0.0001)$, and $6.0(5.0-7.0)(\mathrm{p}<0.0001)$, after 1 month, 3 months and 6 months of treatment with benralizumab, respectively (Figure 1E). These important clinical findings were paralleled by significant improvements in lung function measures. In this regard, in comparison to baseline, $\mathrm{FEV}_{1}$ rose from $1.57 \mathrm{~L}(1.23-2.15)$ to $1.96 \mathrm{~L}(1.60-2.42)(\mathrm{p}<0.0001), 2.09 \mathrm{~L}(1.70-2.53)(\mathrm{p}$ $<0.0001)$, and $2.10 \mathrm{~L}(1.70-2.53)(\mathrm{p}<0.0001)$, after 1 month, 3 months and 6 months, respectively (Figure 1F). FVC increased from a baseline value of 2.47 $\mathrm{L}(2.145-3.055)$ to $2.76 \mathrm{~L}(2.375-3.125)(\mathrm{p}<0.001)$ after 1 month, to $2.94 \mathrm{~L}(2.44-3.415)(\mathrm{p}<0.0001)$ after 3 months, and to $2.91 \mathrm{~L}(2.42-3.43)$ ( $\mathrm{p}<0.0001)$ after 6 months of benralizumab treatment (Figure 1G).

When considering the therapeutic impact on laboratory parameters, it is noteworthy that after 1 month benralizumab zeroed blood eosinophil number, which fell from 740 cells $/ \mu \mathrm{L}(490-1097)$ to 0 cells $/ \mu \mathrm{L}(0-0)(\mathrm{p}<0.0001)$; this result was maintained also after 3 months and 6 months of treatment, respectively (Figure 1H). In 59 out of 111 patients, we also quantified blood basophil number, thus showing that cell count decreased from baseline 46 cells/ $\mu \mathrm{L}(30-90)$ to 6 cells $/ \mu \mathrm{L}(0-20)(\mathrm{p}<0.0001)$ after 1 month, and to 0 cells $/ \mu \mathrm{L}(0-10)(\mathrm{p}<0.0001)$ after 3 months as well as after 6 months, respectively (Figure 1I).

In 39 out of 111 patients, FeNO was also measured at baseline and after 1 month, 3 months, and 6 months of anti-IL-5R $\alpha$ therapy. FeNO levels diminished from baseline values of 48 parts per billion (ppb) (35-67) to $34 \mathrm{ppb}$ $(18-55)(\mathrm{p}<0.01)$ after 1 month, to $30 \mathrm{ppb}(15-48)(\mathrm{p}<$ $0.0001)$ after 3 months, and to $25 \mathrm{ppb}(15-41)$ ( $\mathrm{p}<$ 0.0001 ) after 6 months, respectively (Figure $1 \mathrm{~J})$.

\section{Comparative Evaluation in Relation to Allergic and Non-Allergic Traits}

About the effects of a 6-month treatment with benralizumab on clinical, functional and laboratory indices with respect to baseline, we found some differences between SPT negative and positive patients. The reduction of asthma exacerbation number amounted to $-3(-5$ to -1$)$ in SPT negative patients, and $-5(-7$ to -3$)$ in SPT positive subjects, respectively $(\mathrm{p}<0.01)$ (Figure $2 \mathrm{~A}$ ). In comparison to baseline, the observed increases in ACT score were 5 points (4-9) and 9.5 points (6-13) in patients 
with negative and positive SPT, respectively $(\mathrm{p}<0.01)$ (Figure 2D). Moreover, we detected a decrease in daily SABA use, quantified as -1 inhalations ( -3 to 0 ) in SPT negative group, and -2 inhalations $(-4$ to -1$)$ in SPT positive group $(\mathrm{p}<0.05)$, respectively (Figure $2 \mathrm{C}$ ).

By contrast with the above findings, the following results were not significantly different between the two patient subgroups. Indeed, the decreases of daily OCS intake were $-5 \mathrm{mg}$ ( -10 to 0 ) in patients with negative SPT, and $-5 \mathrm{mg}(-12.5$ to -0$)$ in subjects with positive SPT, respectively $(\mathrm{p}=0.3192)$ (Figure $2 \mathrm{~B})$. AQLQ score increased by 2.5 points (1-3.44) in SPT negative group, and by 2.43 points (1.6-4) in SPT positive group ( $\mathrm{p}=$ 0.4845) (Figure 2E). With respect to baseline, $\mathrm{FEV}_{1}$ increments were $0.43 \mathrm{~L}(0.1625-0.7275)$ in patients with negative SPT, and $0.36 \mathrm{~L}(0.0675-0.9625)$ in subjects with positive SPT $(p=0.697)$ (Figure $2 F)$. FVC increases were $0.17 \mathrm{~L}(0.01-0.57)$ in SPT negative group and 0.34 $\mathrm{L}(0.06-0.7)$ in SPT positive group $(\mathrm{p}=0.7589)$ (Figure $2 \mathrm{G})$. In addition, the decreases in blood eosinophil numbers were -740 cells $/ \mu \mathrm{L}(-1100$ to -520$)$ in patients with negative SPT, and -700 cells/ $\mu \mathrm{L}(-1010$ to -452.5$)$ in subjects with positive SPT $(p=0.6403)$ (Figure $2 \mathrm{H})$. The decrements of blood basophil count were -40 cells $/ \mu \mathrm{L}$ $(-60$ to -20$)$ in SPT negative group, and -44 cells $/ \mu \mathrm{L}$ $(-85$ to -10$)$ in SPT positive group $(\mathrm{p}=0.9243)$ (Figure 2I). Moreover, FeNO level variations were $-26 \mathrm{ppb}(-37.3$ to -15$)$ in patients with negative SPT, and $-17 \mathrm{ppb}(-33$ to 1) in subjects with positive SPT, respectively ( $\mathrm{p}=$ 0.2026) (Figure 2J).

Lastly, we observed no significant correlation between serum IgE concentrations and the 6-month modifications elicited by benralizumab with regard to the following results, including asthma exacerbation reduction $(\mathrm{r}=$ $-0.05876 ; p=0.591$ ) (Figure 3A), OCS intake decrease $(r=-0.06656 ; p=0.5575)$ (Figure 3B), decrement of daily SABA inhalation number $(r=-0.1706 ; p=0.1302)$ (Figure 3C), ACT score increment $(r=0.168 ; p=0.122)$ (Figure 3D), AQLQ score improvement $(\mathrm{r}=0.1388$; $\mathrm{p}=$ 0.3263) (Figure 3E), $\mathrm{FEV}_{1}$ enhancement $(\mathrm{r}=0.06749 ; \mathrm{p}=$ 0.5571) (Figure 3F), FVC increase $(r=0.03773 ; \mathrm{p}=$ 0.7496) (Figure $3 \mathrm{G})$, blood eosinophil decrease $(\mathrm{r}=$ $0.1448 ; \mathrm{p}=0.1808$ ) (Figure $3 \mathrm{H}$ ), blood basophil

Table I Baseline Patient Features

\begin{tabular}{|c|c|c|c|c|}
\hline Characteristics & $\begin{array}{c}\text { Total Population } \mathbf{N}= \\
\text { [ / I }\end{array}$ & $\begin{array}{c}\text { Negative Skin Prick Test } \\
\qquad N=5 I\end{array}$ & $\begin{array}{l}\text { Positive Skin Prick Test } \\
\qquad N=60\end{array}$ & $\mathbf{p}$ \\
\hline Female gender, $\mathrm{N}(\%)$ & $71(63.96)$ & $33(64.7 I)$ & $38(63.33)$ & $>0.9999$ \\
\hline Male gender, $\mathrm{N}(\%)$ & $40(36.04)$ & $18(35.29)$ & $22(36.67)$ & $>0.9999$ \\
\hline Age, median values (IQR), years & $56.00(43.00-65.00)$ & $57.00(49.00-67.00)$ & $51.50(42.25-62.75)$ & 0.0729 \\
\hline $\begin{array}{l}\text { Duration of asthma, median val. } \\
(\mathrm{IQR}) \text {, years }\end{array}$ & $18.0(10.00-30.00)$ & $14.00(10.00-21.00)$ & $24.50(13.00-31.75)$ & 0.0008 \\
\hline FEV & $61.00(49.75-74.25)$ & $66.00(50.00-78.00)$ & $59.00(48.00-73.00)$ & 0.3367 \\
\hline $\begin{array}{l}\text { Blood eosinophils, median v. (IQR), } \\
\text { cells } / \mu \mathrm{L}\end{array}$ & $740.0(200.0-1097)$ & $750.0(550.0-1100)$ & $700.0(452.5-1010)$ & 0.5987 \\
\hline $\begin{array}{l}\text { Blood basophils, median val. (IQR), } \\
\text { cells/ } \mu \mathrm{L}\end{array}$ & $44.0(30.00-86.00)$ & $40.00(20.00-65.00)$ & $46.00(30.00-90.00)$ & 0.7328 \\
\hline BMI, median values (IQR), $\mathrm{kg} / \mathrm{m}^{2}$ & $26.17(23.14-28.13)$ & $25.21(22.84-27.76)$ & $27.02(23.82-28.82)$ & 0.0846 \\
\hline Smokers, N (\%) & $32(28.83)$ & $13(25.49)$ & $19(31.67)$ & 0.5321 \\
\hline $\begin{array}{l}\text { Gastro-esophageal reflux disease, } \\
N(\%)\end{array}$ & $55(49.55)$ & $24(47.06)$ & $31(51.67)$ & 0.7045 \\
\hline Nasal polyposis, N (\%) & $51(45.95)$ & $27(52.94)$ & $24(40.00)$ & 0.1867 \\
\hline Bronchiectasis, N (\%) & $27(24.32)$ & $12(23.53)$ & $15(25.00)$ & $>0.9999$ \\
\hline Osteoporosis, N (\%) & $27(24.32)$ & $12(23.53)$ & $15(25.00)$ & $>0.9999$ \\
\hline Anxiety, N (\%) & $21(18.92)$ & II (2I.57) & $10(16.67)$ & 0.6289 \\
\hline Atopic dermatitis, $\mathrm{N}(\%)$ & $13(11.7 \mid)$ & I (1.96) & $12(20.00)$ & 0.0029 \\
\hline $\begin{array}{l}\text { Obstructive sleep apnea syndrome, } \\
N(\%)\end{array}$ & II (9.91) & $3(5.88)$ & $8(13.33)$ & 0.2204 \\
\hline
\end{tabular}

Note: Bold entries highlight statistically significant differences between patients with negative SPT versus subjects with positive SPT. 

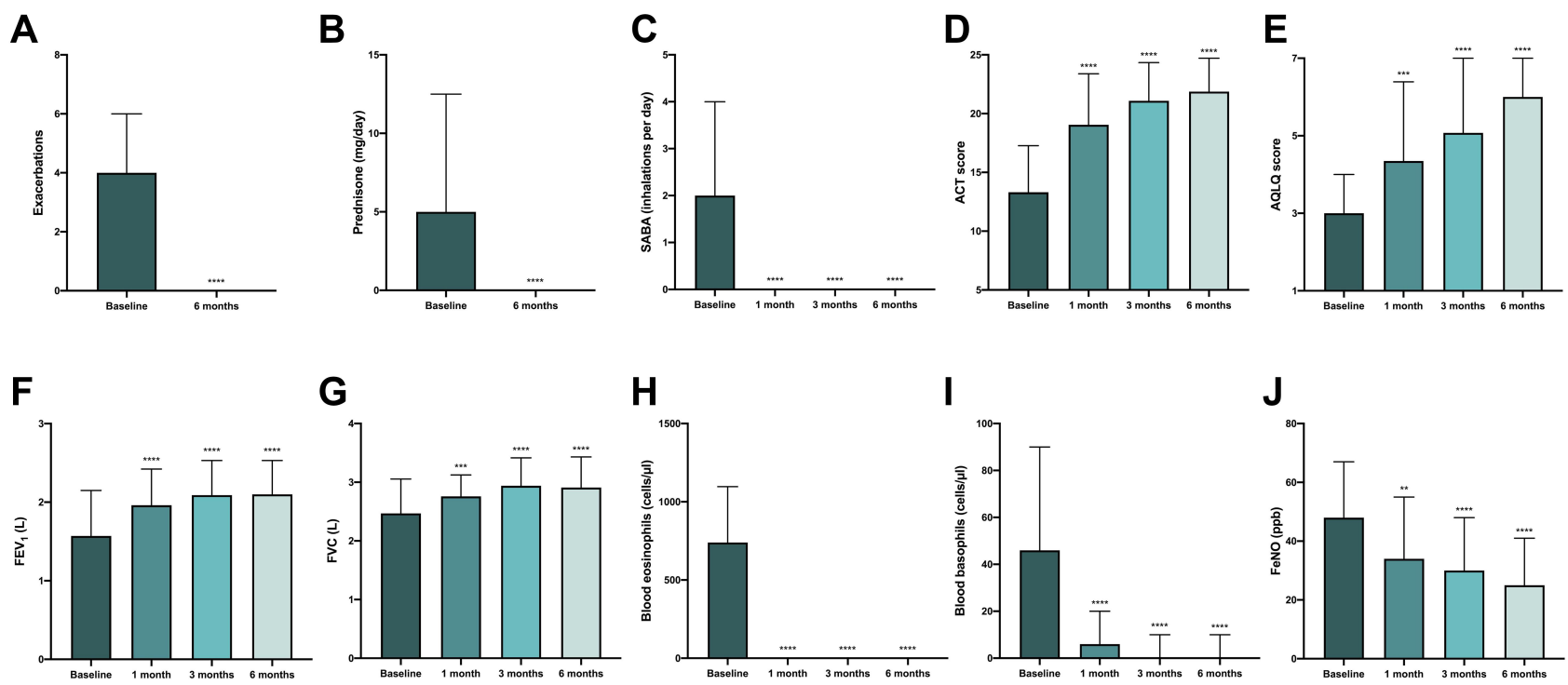

Figure I Efficacy of benralizumab in the whole population of patients with severe persistent eosinophilic asthma, with regard to asthma exacerbation rate (A), prednisone intake (B), daily SABA inhalations (C), ACT score (D), AQLQ score (E), FEV $\mathbf{( F )}$, FVC (G), blood eosinophil numbers (H), blood basophil count (I), and FeNO levels (J). ACT score values are expressed as mean $( \pm S D)$. All other parameters are expressed as median values $(\mathrm{IQR})$. ${ }^{* *} \mathrm{p}<0.0 \mathrm{I} ;{ }^{* * * *} \mathrm{p}<0.00 \mathrm{I}$; ${ }^{* * * *} \mathrm{p}<0.000 \mathrm{I}$.

decrement $(\mathrm{r}=0.06086 ; \mathrm{p}=0.6651)$ (Figure 3I), and FeNO level reduction $(\mathrm{r}=-0.1717 ; \mathrm{p}=0.3026)$ (Figure 3J).

\section{Safety and Tolerability Profile of Benralizumab}

Add-on therapy with benralizumab was well tolerated, and no serious adverse reactions occurred throughout this reallife observational study.

\section{Discussion}

The current multicenter observational study further proves, within a real-life setting, that in patients with severe eosinophilic asthma benralizumab exerts very positive therapeutic effects, already documented by randomized clinical trials, such as SIROCCO and CALIMA. ${ }^{43,44}$ Moreover, our present data confirm, in a quite larger number of severe asthmatic subjects, recent findings referring to realworld investigations carried out in relatively few enrolled patients. $^{33-38}$

In particular, we herein show that the most important result experienced by our patients regarded the marked reduction of asthma exacerbations after 6 months of treatment. Such a relevant anti-asthma action of benralizumab appears to be strictly dependent on eosinophil depletion, which was detected in our patients already after 1 month of treatment. This fast and effective pharmacological activity of benralizumab is likely due to its complex mechanism of action, consisting not only of IL-5R $\alpha$ blockade operated by Fab fragments but also including eosinophil apoptosis induced by antibody-dependent cellmediated cytotoxicity (ADCC). ${ }^{24,25}$ With regard to prevention of asthma exacerbations, it is noteworthy that our current study also showed that benralizumab caused a significant decrease of measured levels of FeNO, another important biomarker of type- 2 inflammation. ${ }^{45}$ However, we analyzed FeNO levels only in 39 out of 111 patients, and benralizumab action was not sufficient to bring FeNO values below $25 \mathrm{ppb}$. Therefore, our findings suggest that in order to assess the overall efficacy of biological therapies targeting either IL-5 or IL-5 receptor, monitoring blood eosinophil count is clinically more relevant than measuring FeNO. Nevertheless, FeNO can represent an accessory inflammatory biomarker in these patients. Indeed, though IL-13 is the main cytokine responsible for inducing the synthesis of NO, the latter can be considered as a surrogate for airway eosinophilic inflammation, ${ }^{46}$ which is thus responsive to the inhibitory action of benralizumab.

It is likely that the observed decrease in asthma exacerbation rate was also favored by noticeable improvements in lung function, expressed as significant $\mathrm{FEV}_{1}$ and FVC increases, which in our real-life context resulted of a greater extent than those reported by the randomized clinical trials SIROCCO, CALIMA and BISE. ${ }^{43,44,47}$ Indeed, worse is the severity of airflow limitation, higher 
A

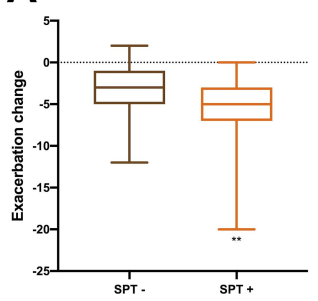

F

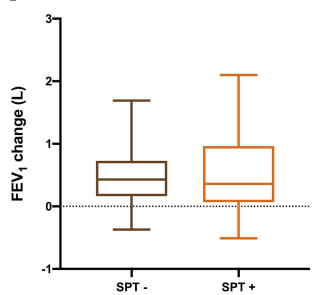

B

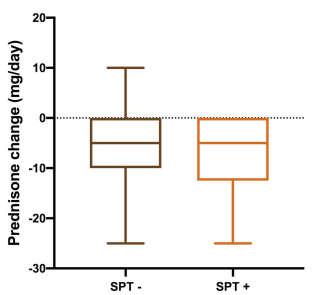

G

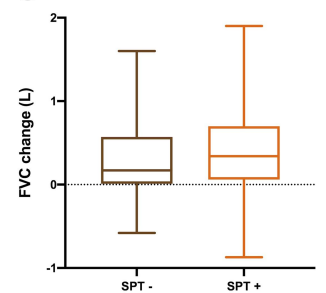

C

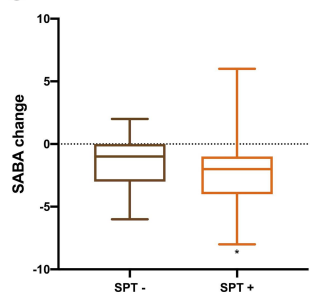

H

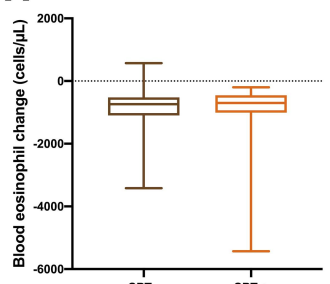

D

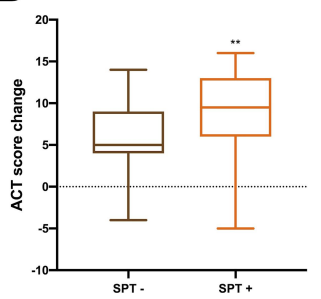

I

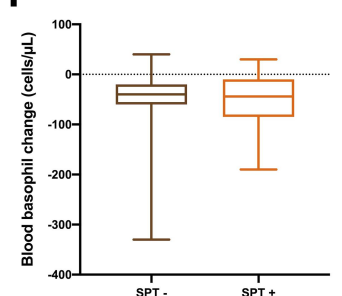

E

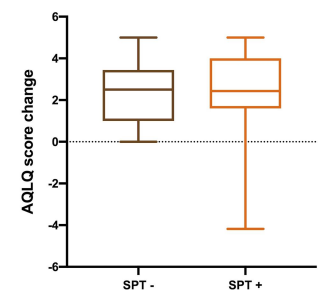

J

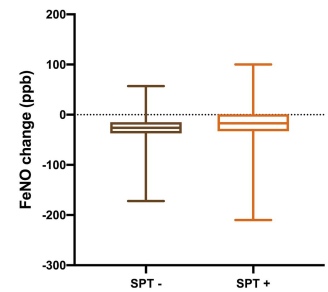

Figure 2 Efficacy of benralizumab in relation to SPT negativity or positivity, with regard to asthma exacerbation rate $(\mathbf{A})$, prednisone intake (B), daily SABA inhalations $(\mathbf{C})$, ACT score (D), AQLQ score (E), FEV $(\mathbf{F})$, FVC (G), blood eosinophil numbers (H), blood basophil count (I), and FeNO levels (J). Boxes display median values and IQR, and whiskers define maximum and minimum. ${ }^{*} \mathrm{p}<0.05 ;{ }^{* *} \mathrm{p}<0.01$.

is the tendency to manifest asthma exacerbations, that in turn promote an acceleration of lung function deterioration. $^{48}$ Therefore, it is reasonable that such a deleterious vicious circle can be effectively interrupted by benralizumab. On the other hand, also benralizumabinduced bronchodilation seems to be dependent on eosinophil depletion because high blood eosinophil counts are associated with severe degrees of bronchial obstruction. ${ }^{8}$ In addition to the eosinophilic inflammatory trait, the atopic status can be another phenotypic feature to be considered when clinicians decide to prescribe benralizumab. Indeed, our results suggest that benralizumab might be even more effective in improving exacerbation rate, as well as in increasing ACT score and decreasing daily SABA use in SPT positive when compared to SPT negative patients.

Asthma exacerbation zeroing, elicited by benralizumab, made it possible for our patients to drastically lower their OCS intake. This real-life effect resulted to be even greater than that one reported by the authors of the
A

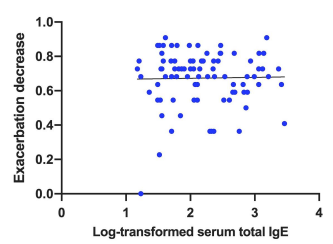

B
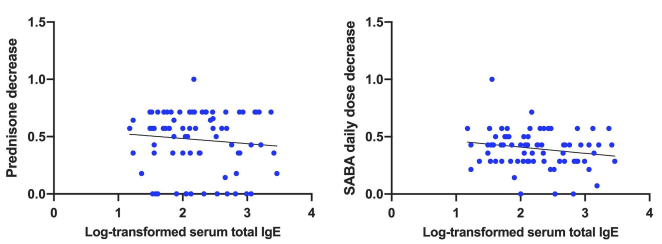

F

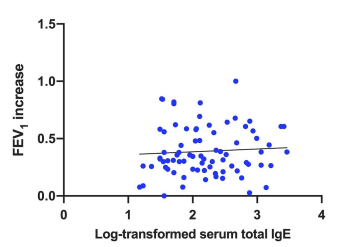

G

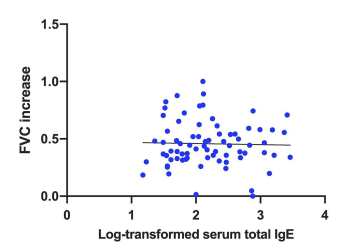

H

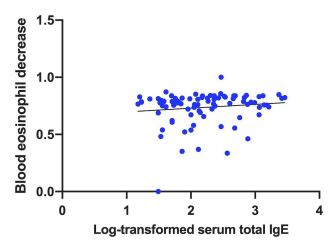

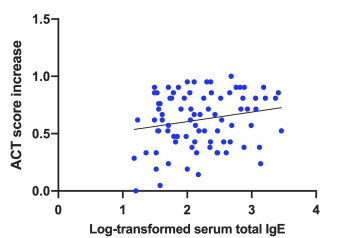

D

I

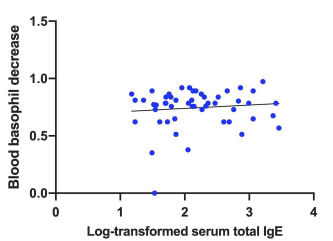

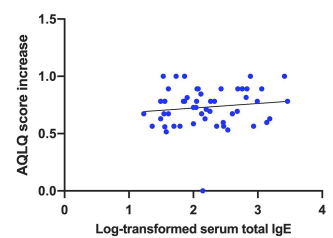

E

\section{J}

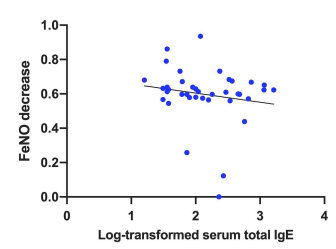

Figure 3 Correlations between serum IgE concentrations, expressed as logarithmic transformation, and 6-month modifications elicited by benralizumab, with regard to asthma exacerbation rate $(\mathbf{A})$, prednisone intake $(\mathbf{B})$, daily SABA inhalations $(\mathbf{C})$, ACT score $(\mathbf{D})$, AQLQ score $(\mathbf{E})$, FEV $(\mathbf{F})$, FVC $(\mathbf{G})$, blood eosinophil numbers $(\mathbf{H})$, blood basophil count (I), and FeNO levels (J). 
ZONDA trial. ${ }^{49}$ The OCS sparing action of benralizumab is clinically relevant, given the frequent occurrence of the well-known potential consequences of OCS therapy (diabetes, hypertension, respiratory infections, osteoporosis and bone fracture, cataract and glaucoma, hypothalamicpituitary-adrenal axis suppression, and decreased growth velocity in children). ${ }^{50}$

In addition to the above-mentioned therapeutic benefits experienced by our patients, benralizumab also improved their quality of life and symptom control, as shown by the significant score increases of AQLQ and ACT, as well as by abrogation of daily SABA assumption. These results, obtained in routine clinical practice, further reinforce the published data referring to SIROCCO and CALIMA phase 3 trials, ${ }^{43,44}$ based on the use of asthma control questionnaire (ACQ)-6 rather than ACT. However, in real-life ACT is perceived by patients as more suitable than ACQ utilized in randomized controlled studies, probably because of a simpler formulation of the various questions. ${ }^{51} \mathrm{We}$ found that after both 3 and 6 months of treatment with benralizumab, ACT score was higher than 20 , which is the reference value considered as indicative of a satisfactory asthma control. ${ }^{52}$

Overall, our results clearly indicate that benralizumab exerted very positive therapeutic effects in both allergic and non-allergic patients with severe eosinophilic asthma. Such findings confirm previous data coming out of a pooled analysis of SIROCCO and CALIMA trials, ${ }^{29}$ as well as of some real-life experiences referring to small numbers of patients. ${ }^{33,35}$ In particular, when considering all benralizumab-induced changes, we detected significant differences in asthma exacerbation rate, ACT score, and daily SABA use between non-allergic and allergic subjects, thus showing that the latter ones experienced better improvements. On the contrary, no significant differences between SPT positive and negative patients were found with regard to the other study outcomes, including changes in OCS intake, AQLQ score, $\mathrm{FEV}_{1}$, FVC, FeNO, and blood eosinophil/basophil counts. Therefore, although ACT improvement was greater in SPT positive versus SPT negative patients, we did not find any difference between these two subgroups with regard to AQLQ. These results suggest that a better symptom control does not necessarily translate into an amelioration of overall quality of life, which likely depends also on other key factors, such as the extent of eosinophilic inflammation, the impairment of respiratory function, and OCS consumption. Indeed, with regard to these three latter aspects, we did not detect any difference between SPT positive and SPT negative subjects. However, this observation should be further confirmed because AQLQ was investigated in only $56(50.5 \%)$ out of the 111 enrolled patients.

The powerful efficacy of benralizumab as add-on therapy of severe eosinophilic, allergic asthma, could be explained on the basis of potential drug interferences with cellular and molecular mechanisms underlying the atopic trait. In fact, elevated levels of IL-5, mature eosinophils and eosinophil progenitors are present in the induced sputum obtained from atopic asthmatic patients. ${ }^{53}$ Besides depleting eosinophils, benralizumab can also elicit inhibitory effects on basophils, which express IL-5R $\alpha .{ }^{54}$ Indeed, in the present real-world investigation we analyzed the numbers of blood basophils in 59 (53.2\%) out of the enrolled 111 study patients, thereby showing that these cell counts dropped after 1 month of benralizumab treatment, and zeroed after 3 and 6 months, respectively. By triggering the apoptosis of basophils, ${ }^{55}$ benralizumab can eliminate a relevant cellular source of IL-4, the key cytokine responsible for Th2 cell commitment and IgE production. ${ }^{12,56}$ Moreover, benralizumab can also lower the number of other cells expressing IL-5R $\alpha$ and producing Th2 cytokines (IL-4, IL-5, IL-13) such as ILC2,${ }^{57}$ which play a pivotal role in the complex crosstalk between innate and adaptive immune responses, underpinning the development and amplification of allergic endotypes. ${ }^{58}$ Benralizumab efficacy in atopic patients has also been recently confirmed by a report of our group, regarding the impressive therapeutic actions of this drug at level of upper and lower airways of patients with severe eosinophilic asthma and allergic nasal polyposis. ${ }^{59}$

Finally, we did not observe any correlation between serum IgE concentrations and the real-life positive outcomes detected in our severe asthmatic patients during treatment with benralizumab. Hence, also these findings corroborate the results of recent pooled analyses of SIROCCO and CALIMA trials, which showed that the therapeutic effects of benralizumab were independent of IgE levels and atopic trait in subjects with severe eosinophilic asthma. ${ }^{29,60}$

In conclusion, despite the typical limitations of reallife studies such as the absence of randomization protocol and placebo control, our multicenter observational investigation clearly indicates that in patients with severe eosinophilic asthma benralizumab was very effective in improving several clinical, functional, and laboratory 
parameters. Within such a context, the most relevant achievable outcome of a 6-month benralizumab treatment was the complete prevention of asthma exacerbations. Benralizumab efficacy was evident in both allergic and non-allergic patients. In particular, when compared to subjects with negative SPT, patients with positive SPT experienced significantly better results with regard to changes in exacerbation rate, ACT score and daily SABA use. However, no correlation was found between study outcomes and serum IgE levels. Taken together, these real-world data further confirm that benralizumab can be a valuable therapeutic choice as add-on biological therapy for patients with severe eosinophilic asthma, characterized by either SPT positivity or negativity. SPT positivity could even provide a further clinical advantage with regard to the improvement in asthma control, elicited by benralizmab.

\section{Acknowledgments COLLABORATORS:}

Raffaele Campisi from Department of Clinical and Experimental Medicine, University of Catania - Catania, Italy.

Danilo Di Bona from Allergology and Clinical Immunology Unit, University "Aldo Moro" of Bari Bari, Italy.

Aikaterini Detoraki from Allergology and Immunology Unit, University "Federico II" of Naples - Naples, Italy.

Carmen Durante and Stefania Principe from Department of Biomedicine and Internal and Specialistic Medicine, University of Palermo - Palermo, Italy.

Maria Pia Foschino Barbaro and Carla Maria Irene Quarato from Department of Medical and Surgical Sciences, Institute of Respiratory Diseases, University of Foggia - Foggia, Italy.

Pietro Impellizzeri from Allergology and Pulmonology Unit, Provincial Outpatient Center of Palermo - Palermo, Italy.

Angelantonio Maglio, Massimo Triggiani, and Carolina Vitale from Department of Medicine, Surgery and Dentistry, University of Salerno - Salerno, Italy.

\section{Authorship Contribution}

All authors contributed to data analysis, drafting or revising the article, have agreed on the journal to which the article will be submitted, gave final approval of the version to be published, and agree to be accountable for all aspects of the work.

\section{Funding}

This research received no specific grant from any funding agency in the public, commercial, or not-for-profit sectors.

\section{Disclosure}

The authors declare that there is no conflict of interest.

\section{References}

1. Stern J, Pier J, Litonjua AA. Asthma epidemiology and risk factors. Semin Immunopathol. 2020;42(1):5-15. doi:10.1007/s00281-020-00785-1

2. Holgate ST, Wenzel S, Postma DS, Weiss ST, Renz H, Sly PD. Asthma. Nat Rev Dis Primers. 2015;1:15025.

3. Pelaia G, Vatrella A, Busceti MT, et al. Cellular mechanisms underlying eosinophilic and neutrophilic airway inflammation in asthma. Mediators Inflamm. 2015;2015:879783. doi:10.1155/2015/879783

4. Papi A, Brightling C, Pedersen SE, Reddel HK. Asthma. Lancet. 2018;391(10122):783-800. doi:10.1016/S0140-6736(17)33311-1

5. Khalaf K, Paoletti G, Puggioni F, et al. Asthma from immune pathogenesis to precision medicine. Semin Immunol. 2019;46:101294. doi:10.1016/j.smim.2019.101294

6. Schoettler N, Strek ME. Recent advances in severe asthma: from phenotypes to personalized medicine. Chest. 2020;157(3):516-528. doi:10.1016/j.chest.2019.10.009

7. McDowell PJ, Heaney LG. Different endotypes and phenotypes drive the heterogeneity in severe asthma. Allergy. 2020;75(2):302-310. doi:10.1111/all.13966

8. Bousquet J, Chanez P, Lacoste JY, et al. Eosinophilic inflammation in asthma. $N$ Engl J Med. 1990;323(15):1033-1039. doi:10.1056/ NEJM199010113231505

9. Nelson RK, Bush A, Stokes J, Nair P, Akuthota P. Eosinophilic asthma. $J$ Allergy Clin Immunol Pract. 2020;8(2):465-473. doi:10.1016/j.jaip.2019.11.024

10. Heffler E, Blasi F, Latorre M, et al. The severe asthma network in Italy: findings and perspectives. J Allergy Clin Immunol Pract. 2019;7(5):1462-1468. doi:10.1016/j.jaip.2018.10.016

11. Schleich F, Brusselle G, Louis R, et al. Heterogeneity of phenotypes in severe asthmatics. The Belgian severe asthma registry (BSAR). Respir Med. 2014;108(12):1723-1732. doi:10.1016/j.rmed.2014.10.007

12. Kuruvilla ME, Lee FE-H, Lee GB. Understanding asthma phenotypes, endotypes, and mechanisms of disease. Clin Rev Allergy Immunol. 2019;56(2):219-233. doi:10.1007/s12016-018-8712-1

13. Tran TN, Zeiger RS, Peters SP, et al. Overlap of atopic, eosinophilic, and TH2-high asthma phenotypes in a general population with current asthma. Ann Allergy Asthma Immunol. 2016;116(1):37-42. doi:10.1016/j.anai.2015.10.027

14. Dunican EM, Fahy JV. Asthma and corticosteroids: time for a more precise approach to treatment. Eur Respir J. 2017;49(6):49. doi:10.1183/13993003.01167-2017

15. Barnes PJ. Corticosteroid resistance in patients with asthma and chronic obstructive pulmonary disease. J Allergy Clin Immunol. 2013;131(3):636-645. doi:10.1016/j.jaci.2012.12.1564

16. Pelaia C, Vatrella A, Crimi C, Gallelli L, Terracciano R, Pelaia G. Clinical relevance of understanding mitogen-activated protein kinases involved in asthma. Expert Rev Respir Med. 2020;14(5):501-510. doi:10.1080/17476348.2020.1735365

17. Global Initiative for Asthma, 2020 update. Available from: www. ginasthma.org. Accessed February 12, 2020.

18. Pelaia C, Calabrese C, Terracciano R, de Blasio F, Vatrella A, Pelaia G. Omalizumab, the first available antibody for biological treatment of severe asthma: more than a decade of real-life effectiveness. Ther Adv Respir Dis. 2018;12:1753466618810192. doi:10.1177/1753466618810192 
19. Pelaia C, Calabrese C, Barbuto S, et al. Omalizumab lowers asthma exacerbations, oral corticosteroid intake and blood eosinophils: results of a 5-YEAR single-centre observational study. Pulm Pharmacol Ther. 2019;54:25-30. doi:10.1016/j.pupt.2018.11.002

20. Pelaia C, Vatrella A, Busceti MT, et al. Severe eosinophilic asthma: from the pathogenic role of interleukin-5 to the therapeutic action of mepolizumab. Drug Des Devel Ther. 2017;11:3137-3144. doi:10.2147/DDDT.S150656

21. Pelaia C, Busceti MT, Solinas S, Terracciano R, Pelaia G. Real-life evaluation of the clinical, functional, and hematological effects of mepolizumab in patients with severe eosinophilic asthma: results of a single-centre observational study. Pulm Pharmacol Ther. 2018;53:1-5. doi:10.1016/j.pupt.2018.09.006

22. Sposato B, Camiciottoli G, Bacci E, et al. Mepolizumab effectiveness on small airway obstruction, corticosteroid sparing and maintenance therapy step-down in real life. Pulm Pharmacol Ther. 2020;61:101899. doi:10.1016/j.pupt.2020.101899

23. Pelaia G, Vatrella A, Busceti MT, et al. Role of biologics in severe eosinophilic asthma - focus on reslizumab. Ther Clin Risk Manag. 2016;12:1075-1082. doi:10.2147/TCRM.S111862

24. Pelaia C, Vatrella A, Bruni A, Terracciano R, Pelaia G. Benralizumab in the treatment of severe asthma: design, development and potential place in therapy. Drug Des Devel Ther. 2018;12:619-628. doi:10.2147/DDDT.S155307

25. Pelaia C, Calabrese C, Vatrella A, et al. Benralizumab: from the basic mechanism of action to the potential use in the biological therapy of severe eosinophilic asthma. Biomed Res Int. 2018;2018:4839230. doi: 10.1155/2018/4839230

26. Pelaia C, Vatrella A, Gallelli L, et al. Dupilumab for the treatment of asthma. Expert Opin Biol Ther. 2017;17(12):1565-1572. doi:10.1080/14712598.2017.1387245

27. Albers FC, Müllerová H, Gunsoy NB, et al. Biologic treatment eligibility for real-world patients with severe asthma: the IDEAL study. J Asthma. 2018;55(2):152-160. doi:10.1080/02770903.2017.1322611

28. Humbert M, Albers FC, Bratton DJ, et al. Effect of mepolizumab in severe eosinophilic asthma according to omalizumab eligibility. Respir Med. 2019;154:69-75. doi:10.1016/j.rmed.2019.06.004

29. Chipps BE, Newbold P, Hirsch I, Trudo F, Goldman M. Benralizumab efficacy by atopy status and serum immunoglobulin E for patients with severe, uncontrolled asthma. Ann Allergy Asthma Immunol. 2018;120(5):504-511.e4. doi:10.1016/j.anai.2018.01.030

30. Corren J, Castro M, O'Riordan T, et al. Dupilumab efficacy in $\mathrm{P}$ patients with uncontrolled, moderate-to-severe allergic asthma. J Allergy Clin Immunol Pract. 2020;8(2):516-526. doi:10.1016/j. jaip.2019.08.050

31. Pelaia C, Crimi C, Pelaia G, et al. Real-life evaluation of mepolizumab efficacy in patients with severe eosinophilic asthma, according to atopic trait and allergic phenotype. Clin Exp Allergy. 2020;50 (7):780-788. doi:10.1111/cea.13613

32. Carpagnano GE, Pelaia C, D'Amato M, et al. Switching from omalizumab to mepolizumab: real-life experience from Southern Italy. Ther Adv Respir Dis. 2020;14:1753466620929231. doi:10.1177/ 1753466620929231

33. Pelaia C, Busceti MT, Vatrella A, et al. Real-life rapidity of benralizumab effects in patients with severe allergic eosinophilic asthma: assessment of blood eosinophils, symptom control, lung function and oral corticosteroid intake after the first drug dose. Pulm Pharmacol Ther. 2019;58:101830. doi:10.1016/j.pupt.2019.101830

34. Pelaia C, Busceti MT, Vatrella A, et al. Effects of the first three doses of benralizumab on symptom control, lung function, blood eosinophils, oral corticosteroid intake, and nasal polyps in a patient with severe allergic asthma. SAGE Open Med Case Rep. 2020;8:2050313X20906963. doi:10.1177/2050313X20906963
35. Pelaia C, Busceti MT, Crimi C, et al. Real-life effects of benralizumab on exacerbation number and lung hyperinflation in atopic patients with severe eosinophilic asthma. Biomed Pharmacother. 2020;129:110444. doi:10.1016/j.biopha.2020.110444

36. Di Bona D, Minenna E, Albanesi M, Nettis E, Caiaffa MF, Macchia L. Benralizumab improves patient reported outcomes and functional parameters in difficult-to-treat patients with severe asthma: data from a real-life cohort. Pulm Pharmacol Ther. 2020;64:101974. doi:10.1016/j.pupt.2020.101974

37. Kavanagh JE, Hearn AP, Dhariwal J, et al. Real-world effectiveness of benralizumab in severe eosinophilic asthma. Chest. 2020;158(2). doi:10.1016/j.chest.2020.03.042.

38. Padilla-Galo A, Levy-Abitbol R, Olveira C, et al. Real-life experience with benralizumab during 6 months. BMC Pulm Med. 2020;20 (1):184. doi:10.1186/s12890-020-01220-9

39. Hentges F, Léonard C, Arumugam K, Hilger C. Immune responses to inhalant mammalian allergens. Front Immunol. 2014;5:234. doi:10.3389/fimmu.2014.00234

40. Froidure A, Mouthuy J, Durham SR, Chanez P, Sibille Y, Pilette C. Asthma phenotypes and IgE responses. Eur Respir J. 2016;47 (1):304-319. doi:10.1183/13993003.01824-2014

41. Chung KF, Wenzel SE, Brozek JL, et al. International ERS/ATS guidelines on definition, evaluation and treatment of severe asthma. Eur Respir J. 2014;43(2):343-373. doi:10.1183/ 09031936.00202013

42. Graham BL, Steenbruggen I, Miller MR, et al. Standardization of spirometry 2019 update. An official American Thoracic Society and European Respiratory Society technical statement. Am J Respir Crit Care Med. 2019;200(8):e70-88. doi:10.1164/rccm.201908-1590ST

43. Bleecker ER, FitzGerald JM, Chanez P, et al. Efficacy and safety of benralizumab for patients with severe asthma uncontrolled with highdosage inhaled corticosteroids and long-acting $\beta 2$-agonists (SIROCCO): a randomised, multicentre, placebo-controlled phase 3 trial. Lancet. 2016;388(10056):2115-2127. doi:10.1016/S01406736(16)31324-1

44. FitzGerald JM, Bleecker ER, Nair P, et al. Benralizumab, an antiinterleukin-5 receptor $\alpha$ monoclonal antibody, as add-on treatment for patients with severe, uncontrolled, eosinophilic asthma (CALIMA): a randomised, double-blind, placebo-controlled phase 3 trial. Lancet. 2016;388(10056):2128-2141. doi:10.1016/S01406736(16)31322-8

45. Mogensen I, James A, Malinovschi A. Systemic and breath biomarkers for asthma: an update. Curr Opin Allergy Clin Immunol. 2020;20 (1):71-79. doi:10.1097/ACI.0000000000000599

46. Barnes PJ, Dweik RA, Gelb AF, et al. Exhaled nitric oxide in pulmonary diseases: a comprehensive review. Chest. 2010;138 (3):682-692. doi:10.1378/chest.09-2090

47. Ferguson GT, FitzGerald JM, Bleecker ER, et al. Benralizumab for patients with mild to moderate, persistent asthma (BISE): a randomised, double-blind, placebo-controlled, phase 3 trial. Lancet Respir Med. 2017;5(7):568-576. doi:10.1016/S22132600(17)30190-X

48. Bai TR, Vonk JM, Postma DS, Boezen HM. Severe exacerbations predict excess lung function decline in asthma. Eur Respir J. 2007;30 (3):452-456. doi:10.1183/09031936.00165106

49. Nair P, Wenzel S, Rabe KF, et al. Oral glucocorticoid-sparing effect of benralizumab in severe asthma. $N$ Engl J Med. 2017;376 (25):2448-2458. doi:10.1056/NEJMoa1703501

50. Canonica GW, Colombo GL, Bruno GM, et al. Shadow cost of oral corticosteroids-related adverse events: a pharmacoeconomic evaluation applied to real-life data from the severe asthma network in Italy (SANI) registry. World Allergy Organ J. 2019;12(1):100007. doi:10.1016/j.waojou.2018.12.001 
51. Jia CE, Zhang HP, Lv Y, et al. The asthma control test and asthma control questionnaire for assessing asthma control: systematic review and meta-analysis. J Allergy Clin Immunol. 2013;131(3):695-703. doi:10.1016/j.jaci.2012.08.023

52. Nathan RA, Sorkness CA, Kosinski M, et al. Development of the asthma control test: a survey for assessing asthma control. J Allergy Clin Immunol. 2004;113(1):59-65. doi:10.1016/j.jaci.2003.09.008

53. Dorman SC, Efthimiadis A, Babirad I, et al. Sputum CD34+IL-5R $\alpha+$ cells increase after allergen. Am J Respir Crit Care Med. 2004;169 (5):573-577. doi:10.1164/rccm.200307-1004OC

54. Valent P, Dahinden CA. Role of interleukins in the regulation of basophil development and secretion. Curr Opin Hematol. 2010;17 (1):60-66. doi:10.1097/MOH.0b013e328331fae9

55. Kolbeck R, Kozhich A, Koike M, et al. MEDI-563, a humanized anti-IL-5 receptor alpha $\mathrm{mAb}$ with enhanced antibody-dependen cell-mediated cytotoxicity function. $J$ Allergy Clin Immunol. 2010;125(6):1344-1353.e2. doi:10.1016/j.jaci.2010.04.004

56. Kaiko GE, Horvat JC, Beagley KW, Hansbro PM. Immunological decision-making: how does the immune system decide to mount a helper T-cell response? Immunology. 2008;123(3):326-338. doi:10.1111/j.1365-2567.2007.02719.x
57. Sehmi R, Lim HF, Mukherjee M, et al. Benralizumab attenuates airway eosinophilia in prednisone-dependent asthma. J Allergy Clin Immunol. 2018;141(4):1529-1532.e8. doi:10.1016/j.jaci.2018.01.008

58. Lambrecht BN, Hammad H. The immunology of asthma. Nat Immunol. 2015;16(1):45-56. doi:10.1038/ni.3049

59. Lombardo N, Pelaia C, Ciriolo $M$, et al. Real-life effects of benralizumab on allergic chronic rhinosinusitis and nasal polyposis associated with severe asthma. Int $J$ Immunopathol Pharmacol. 2020;34:2058738420950851. doi:10.1177/ 2058738420950851

60. Jackson DJ, Humbert M, Hirsch I, Newbold P, Garcia Gil E. Ability of serum IgE concentration to predict exacerbation risk and benralizumab efficacy for patients with severe eosinophilic asthma. Adv Ther. 2020;37(2):718-729. doi:10.1007/s12325-01901191-2

\section{Publish your work in this journal}

The Journal of Asthma and Allergy is an international, peer-reviewed open-access journal publishing original research, reports, editorials and commentaries on the following topics: Asthma; Pulmonary physiology; Asthma related clinical health; Clinical immunology and the immunological basis of disease; Pharmacological interventions and new therapies. The manuscript management system is completely online and includes a very quick and fair peer-review system, which is all easy to use. Visit http://www.dovepress.com/testimonials.php to read real quotes from published authors. 